NAL PROPOSAL NO. $\quad$ 24-A

Correspondent: D. O. Caldwel1 Department of Physics University of Calif. Santa Barbara, Calif.

FTS/Off-net: $\quad$ 805-963-3611

$961-2311$

MEASUREMENT OF INELASTIC COMPTON SCATTERING

D. O. Caldwell, V. B. Elings, A. J. Greenberg, B. N. Kendall, R. J. Morrison, and F. V. Murphy University of California at Santa Barbara

M. Atac

National Accelerator Laboratory

June 1970 
Revision

NAL Proposal No. 24

Correspondent: D. O. Caldwell

\title{
MEASUREMENT OF INELASTIC COMPTON SCATTERING
}

\author{
Submitted by
}

D. O. Caldwell, V. B. Elings, A. J. Greenberg, B. N. Kendall,

R. J. Morrison, and F. V. Murphy

University of California at Santa Barbara

and

M. Atac

National Accelerator Laboratory

June 1970 
1. Title of Experiment--Measurement of Inelastic Compton Scattering

2. Experimenters--D. Caldwell, V. Elings, A. Greenberg, B. Kendall, R. Morrison, and F. Murphy

3. Summary of Experiment

Inelastic electron scattering data indicates that the electrons may be scattering from point-like objects in the nucleon. A model by Bjorken, Feynman, and Paschos predicts that there is a relationship between the cross sections for inelastic Compton scattering and inelastic electron scattering which depends on the average charge of the point-like constitutents of the nucleons. We propose, therefore, to measure the inelastic Compton scattering from deuterjum for an incident bremsstrahlung energy of $40 \mathrm{GeV}$.

4. Equipment Required for Experiment

a. A liquid deuterium target with a cell $10 \mathrm{~cm}$ in diameter and $1 \mathrm{~m}$ in length.

b. A. 240-in. H magnet capable of $18 \mathrm{kG}$.

5. Beam Requirements

Three-hundred hours of "prime time" with $4 \times 10^{7}$ electrons/pulse at $40 \mathrm{GeV}$ for data taking and equipment checkout; 100 hours of lowpriority time for checkout of the equipment.

6. Time Ready

We could be ready for checkout when the machine turns on. 
THEORETICAL MOTIVATION FOR THE FXPERIMENT

Deep inelastic electron scattering experiments at SLAC have shown that cross sections beyond the resonance region are very large and decrease much more slowly with momentum transfer than do the cross sections for either elastic scattering or production of specific resonances. Theoretical speculations, therefore, have focused on the possibility that these data might indicate point-ljke charged structures within the nucleon.

To review the situation more quantitatively, the cross section for inelastic electron scattering can be written ${ }^{1}$

$$
\frac{d^{2} \sigma}{d \Omega d E^{\prime}}=\frac{\alpha^{2}}{4 E^{2} \sin ^{4} \theta / 2}\left[W_{2} \cos ^{2} \theta / 2+2 W_{1} \sin ^{2} \theta / 2\right]
$$

where

$$
\begin{aligned}
& E=\text { energy of incident electron, } \\
& E^{\prime}=\text { energy of scattered electron, } \\
& \theta=\text { angle of scattered electron, } \\
& v=\mathrm{E}-\mathrm{E}^{\prime} .
\end{aligned}
$$

$W_{1}\left(q^{2}, v\right)$ and $W_{2}\left(q^{2}, v\right)$ are relativistically invariant functions which describe the nucleon's properties.

At high energies and large momentum transfers, the cross section can be written as 


$$
\frac{\mathrm{d}^{2} \sigma}{\mathrm{d} \Omega \mathrm{dE}}=\frac{\alpha^{2} W_{2}}{4 \mathrm{E}^{2} \sin ^{4} \theta / 2}\left[1+\frac{v^{2}}{2 \mathrm{EE}} \frac{\sigma_{t}}{\sigma_{t}+\sigma_{\ell}}\right] \text {, }
$$

where $\sigma_{t}$ and $\sigma_{\ell}$ are proportional to the cross sections for transverse and longitudinally polarized photons. For moderate inelasticities and high incident energies, the second term in the parenthesis is small for the entire region $0<\sigma_{t} / \sigma_{\ell}<\infty$.

What the inelastic electron scattering has shown is that $v W_{2}$ is a function only of $v / q^{2}$. The experimental results are shown in Fig. 1. As one can see, the function $v \mathrm{~W}_{2}$ approaches a constant for $v / \mathrm{q}^{2}$ $\geq 2 \mathrm{BeV}^{-1}$.

Bjorken, Feynman, and Paschos ${ }^{1,2}$ have proposed a model to explain the striking results of the inelastic electron scattering. Their model assumes that the nucleon is composed of point-like charged objects which they call partons. They argue that the inelastic electron scattering, then, can be understoød in terms of the electron's scattering incoherently from these individual constitutents of the nucleon. They tried to fit the electron data assuming that the proton consisted of throe quarks with the usual charges. They find that in this case $v W_{2}$ is a rapidly varying function of $v / q^{2}$ and goes to zero in the limit $v / q^{2} \rightarrow \infty$, which does not agree with the data. They also tried a model which assumes that besides the three quarks there is an infinite sea of quark; anti-quark pairs. With this particular composition they find that they can make a reasonable fit to the data. 
Bjorken and Paschos have shown ${ }^{1}$ that independent of the details of the model, there is a simple relation between the cross section for inelastic Compton scattering and the cross section for inelastic electron scattering

$$
\left.\frac{d^{2} \sigma}{d \Omega d E^{\prime}}\right|_{\gamma p}=\left.\frac{\nu^{2}}{E E^{\prime}} \quad \frac{d^{2} \sigma}{d \Omega d^{1}}\right|_{\text {ep }} \frac{\left\langle Q^{4}\right\rangle}{\left\langle Q^{2}\right\rangle},
$$

where $\left\langle Q^{n}\right\rangle$ is the nth power of the charge of the constituents (partons) averaged over the constituents.

If the partons have integer charge, $\left\langle Q^{4}\right\rangle /\left\langle Q^{2}\right\rangle=1$. For the three-quark model in an infinite sea of quark, anti-quark pairs, they have calculated upper and lower limits which are

$$
\frac{1}{3}<\frac{\left\langle Q^{4}\right\rangle}{\left\langle Q^{2}\right\rangle}<\frac{5}{9} .
$$

Thus in this model the ratio of photon to electron inelastic scattering would provide a measure of the charge of the point-like objects doing the scattering.

R.: Blankenbecler has conjectured ${ }^{3}$ that the electron scattering data might be understood in terms of the electron scattering from nonpoint-like objects with form factors (such as pions) in which the contributions of the possible final states increase with momentum transfer in such a way that the inelastic cross section is independent of the momentum transfer. This point of view is implicit in the field theoretical 
basis of the parton model as developed by Drell and co-workers. In a model such as this, one might expect the Compton yield to be higher than that calculated by Bjorken and Paschos, since the form factors of the non-point-like objects would be higher for real incident photons (Compton scattering) than for virtual photons (electron scattering).

A third model probably giving the sort of results observed in inelastic electron scattering is the strong-coupling model in the form developed by A. Krass. ${ }^{4}$ Because this is equivalent to having one point particle within the nucleon, the photon to electron inelastic scattering cross sections would have a ratio similar to the one given by Bjorken and Paschos but with $Q=1$.

In general, it appears likely that highly inelastic Compton scattering of appreciable cross section may exist. Its magnitude and momentum-transfer dependence, if observed, may then serve as a means of choosing among several models.

The main problem with measuring the inelastic Compton scattering cross section is that the scattered photon must be observed in a background of photons coming from the decay of photoproduced $\pi^{\circ} \mathrm{s}$. Using equipment similar to that proposed for this experiment, our group has already made an attempt to measure the inelastic Compton scattering at SLAC with a 20-GeV incident bremsstrahlung beam. Figure 2 shows the calculated yields of $\gamma^{\prime}$ s from Compton scattering and the decay of $\pi^{\circ}$ 's for a $20-\mathrm{GeV}$ bremsstrahlung beam. It is clear that even at the 
most favorable angle and energy for the scattered $\gamma$, the separation of the two processes would be difficult. Our data indicates that the yield of $\gamma^{\prime}$ s from $\pi^{\circ}$ decay is equal to that which we expected; i.e., the yield of $\pi^{\circ}$ s was equal to the average of the $\pi^{+}$and $\pi^{-}$yields which were measured in the beam survey at SLAC. Whether or not our data indicates any inelastically Compton scattered photons is not yet clear.

If one uses a 40-GeV incident bremsstrahlung beam, as we propose in this experiment, the situation changes dramatically for the better. Figure 3 shows, in this case, the calculated yield of $\gamma^{\prime}$ s from inelastic Compton scattering and the decay of $\pi^{\circ} / \mathrm{s}$ at various angles and energies. The Compton yield was calculated from relationship (3) assuming a parton charge of one. The $\pi^{\circ}$ yield was calculated using a . multi-Regge exchange model ${ }^{5}$ to scale up to $40 \mathrm{GeV}$ the average of the $\pi^{+}$and $\pi^{-}$yields measured as SLAC with an 18 -GeV bremsstrahlung beam. At scattered $\gamma$ energies of $20 \mathrm{GeV}$ and angles of $6^{\circ}$, the inelastic Compton yield is about 100 timaes the $\pi^{\circ}$ decay yield, a vast improvernent over the situation at SIAC energies. In addition, the high duty cycle available at NAL will make possible the reduction and meas urement of this background.

We feel that the inelastic Compton scattering experiment is a very important one and asks basic questions about the make-up of nucleons. This measurement will not be possible at Serpukhov as the photon intensities are too low. The proton intensity is down by $10^{-2}$ from NAL, 6 
and we have calculated that there is another factor of $10^{-2}$ in photon production due to the lower proton energy, giving a photon intensity $10^{4}$ lower than is possible at NAI in the energy range 20-40 GeV. The experimental setup which we propose in the next section is quite simple and inexpensive but has proven to operate very well. at SLAC under much more severe conditions than will exist at NAL. For the cost involved, compared to the physics which can develop from this experiment, we feel that it should certainly be one of the first experiments to run at NAL. One of us (Rollin Morrison) will, while attending the 1970 NAI Summer Study, look into the details involved in modifying the 3.5-mrad beam so that it can be used as an electron beam.

\section{EXPERIMENTAL ME'THOD}

The experimental method which we propose is quite simple. It consists of running a $40-\mathrm{GeV}$ bremsstrahlung beam into a liquid deuterium datget and looking, with an array of $40 \mathrm{~Pb}$-glass total absorption shower counters, for wide-angle photons emitted from the target. The experimental arrangement is shown in Fig. 4.

- The bremsitrahlung beam is produced by an electron beam im pinging on a $0.2 \mathrm{r} .1$. radiator. The electron beam is described in our proposal for the measurement of the total $\gamma$-nucleus cross section and in the summer-study reports of Toner ${ }^{7}$ and Diebold and Hand. ${ }^{8}$ The yjeld of electrons expected for the beam is shown in Fig. 5. In order to obtain an acceptable counting rate, we have chosen to run at an electron energy of $40 \mathrm{GeV}$. 
After the electrons pass through the radjator, they are bent by a 240-in. magnet into a beam dump. The experiment has been designed with the magnet operating at $9 \mathrm{kG}$ so that the experiment can be easily extended to electron energies of $80 \mathrm{GeV}$ if the results warrant it. The photons continue forward into a 1-meter-long target'which contains deutexium. Photons which do not interact in the target and $e^{+} e^{-}$pairs produced in the target go forward into a quantameter which measures the beam flux.

One of the concerns in any photon experiment at NAL will be the effects of pion contamination of the electron beam. This has been estimated by Diebold and Hand ${ }^{8}$ to be less than $10^{-3}$ of the electron flux. One can then expect that for each $10^{4}$ photons produced in the upper $30 \%$ of the bremsstrahlung spectrum there will be $\sim 1$ pion interaction in the radiator. Neutral hadrons could pass through the magnet and have a high probability of interacting in the liquid target. Such events would

- be less than an upper limit of $2.0 \%$ of the photon-induced events.

This contamination will be reduced by a factor of at least 100 by the introduction of veto counters shown in Fig. 4. The hadron detector upstream of the magnet, is constructed from several lead-scintillator layers which will detect at least one particle from a hadron interaction in the radiator. This is essentially the hadron detector used in our total cross-section measurement. "The beam hole will be chosen such that bremsstrahlung and pairs produced in the radiator, cheracterized 
by small production angles, will pass through while hadrons produced with angles $\sim 400 \mathrm{MeV} / \mathrm{E}$ will be detected. The veto counters $\mathrm{A}_{1}, \mathrm{~A}_{2}$, and $A_{0}$ will also help with the discrimination against pion-induced events. The beam-defining shower veto counter $A_{0}$ will also protect agajnst wide-angle bremsstrahlung. If the photons are tagged with shower counters, as described in our total cross-section proposal, this would provide another factor of 10-100 in discrimination.

Photons which are inelastically Compton scattered (and those from $\pi^{\circ}$ decays) are detected by an array of 40 Pb-glass shower counters which are at a distance of $5 \mathrm{~m}$ from the target. Each shower counter consists of a $2 \frac{1}{2}-i n . \times 2 \frac{1}{2}-$ in. $\times 13 \frac{1}{2}-$ in. Pb-glass block $(50 \% \mathrm{~Pb}$ by weight, radiation length $=1$ in.) with a 2 -in. phototube optically coupled to the rear. The blocks are stacked to form a $5 \times 8$ array as shown in Fig. 4. The array subtends $\gamma$ scattering angles from $6^{\circ}$ to $12^{\circ}$ and has an angular resolution of $\pm 6 \mathrm{mrad}\left( \pm 0.37^{\circ}\right)$. There is good pos sibility that by the time this experiment is run, our group will obtain an additional 24 blocks, making a total of 64 . This will increase the proposed counting rates by $-50 \%$. The $\mathrm{Pb}-$ glass counters, which we have already used in experiments at SLAC and LRI, have a resolution of $6 \%$ fwhm for photon energies of $12 \mathrm{GeV}$. This resolution improves with energy, going as $1 / \sqrt{\mathrm{E}} \gamma^{\prime}$ The phototubes on each of the Pb-glass counters are connected to sample-and hold circuits which in turn feed analog-to-digital converters. For every event the pulse heights from 
each of the 40 counters is digitized and read into our PDP-15 computer which will be operating on-line.

In addition to single photons, the lead-glass array has a high probability for detecting both of the photons from $\pi^{\circ}$ decays. The opening angle for a $20-\mathrm{GeV} \pi^{\circ}$ corresponds to about the width of one lead-glass block in the geometry shown in Fig. 4. To reduce the con.fusion due to photons striking between the blocks, we plan to improve the spatial resolution by converting the photons in front of the lead glass in $2-3$ radiation lengths of lead followed by proportional planes. We have made tests which show that $2-3$ radiation lengths of material in front of a shower counter do not significantly worsen the resolution of that counter.

The information from the proportional planes will help not only in measuring the $\pi^{\circ}$ background but also in reducing the background on an event by event basis. Due to the steep slope of the $\pi^{\circ}$ energy distribution, the photons observed at a given energy tend to come from

- asymmetric decays from $\pi^{0}$ s of only slightly higher energy. The pro-. bability of detecting both photons from such decays is hard to estimate since it will depend upon experimental conditions. The use of proportional planes in conjunction with shower detectors is, as far as we know, an untried technique. The additional information from these detectors will make the experiment a bit cleaner but is not essential to its success. Because of the low counting ratc for high-energy secondary $\gamma$ 
rays, (on the order of tens of counts/hour), it is important that one eliminate background events. In order to insure that large pulses in the shower counters are accompanied by an interaction in the target, we propose to trigger the electronics of a signal from a counter placed downstream of the target, as shown in Fig. 1. This counter is designed to detect hadrons or their decay products emitted from the target. The counter is constructed of 4 alternate layers of $1 \frac{1}{2}$-in. thick lead and $\frac{1}{2}$ in. thick scintillator. We have used such a counter in our total $\gamma$ nucleon cross-section measurements at SI.AC and found it to be more than $99 \%$ efficient for detecting hadrons emerging from the target. The detector will be insensitive to low-energy below $1 \mathrm{GeV}$ ) electromagnetic background and, therefore, the rate in the counter will not be excessively high.

Another way to obtain a trigger would be to tag the photon beam. Although this is feasible, it would require another magnet. Our philosophy is to make the experiment as simple and inexpensive to NAL as possible, but still have an apparatus which will do the job. If our other proposal (to measure the total photoabsorption cross sectien) is ap proved, then we could use that setup, with its tagging system, to perform this experiment. The only changes in the setup that would be required are the installation of a thicker radiator, the installation of the $\mathrm{Pb}$-glass array, and the remodeling of the holc in the hadron counter, $\mathrm{s}_{2}$, to allow a clear path from the target to the Pb-glass array. 
$\mathrm{S}_{2}$ would then be used in coincidence with the tagging counter to provide a trigger. The tagging system and the shower counter $\mathrm{S}_{1}$ would both serve as beam monitors.

The tagging system has two additional advantages. In the first place it gives the incoming photon energy so that one would not have to fold the bremsstrahlung spectrum into the parton model as has been done in Figs. 2 and 3. In addition, the tagging system provides a large additional factor of discrimination against events related to the pion beam contamination.

The expected yields in 5 of the $40 \mathrm{~Pb}$-glass counters for 200 hours of running time using a $1-\mathrm{m}$ long $\mathrm{D}$ target are shown in Fig. 6 . Figure 6 shows 3 curves: 1 ) the $\gamma$ yield expected if there is no inelastic Compton yield and if all of the $\gamma^{\prime}$ s come from the decay of $\pi^{\circ} s$ which are photoproduced in the target. The $\pi^{\circ}$ yield was calculated in the manner dis cussed previousiy. 2) The yield of $\gamma^{\prime}$ s expected from both $\pi^{\circ}$ decay and inelastic Compton scattering using the model of Bjorken and Paschos with partons of unit charge $(Q=1)$. 3) The same as 2 but with partons of $\left\langle Q^{4}\right\rangle \mid\left\langle Q^{2}\right\rangle=1 / 3$ which is the least favorable possibility if the par tons are quarks. The yields in Fig. 6 are only for the one column of $\mathrm{Pb}$-glass counters subtending scattering angles between 6.8 and $6.75^{\circ}$, i. e. only 5 of the 40 counters. Similar data would be collected simultaneously by the other 35 counters in the array.

- It should be emphasized that the cross section at which the inelastic 
Compton yield is equal to the yield from the decay of $\pi^{\circ} \mathrm{s}$ is almost independent of angle. As shown in Fig. 3, the crossover point is at $\mathrm{d}^{2} \sigma / \mathrm{d} \Omega \mathrm{dk}=10^{-32} \mathrm{~cm}^{2} / \mathrm{sr} \mathrm{GeV}$. This means that all eight eolumns of counters will be equally sensitive in detecting an inelastic Compton yield against the background of gammas from $\pi^{\circ}$ decays.

The shower counters will be calibrated by turning off the ditching magnet and moving the counter array into the beam line so that the electrons enter directly into the $\mathrm{Pb}$ glass. Knowing the energy of the electrons, the pulse heights from the counters can be calibrated.

All counters and electronics, including the PDP-15 computer, will be supplied by our group.

\section{RUNNING TIME RIQQUTPED}

As shown in Fig. 6, one can determine in 200 hours of running time if there is an inelastic Compton scattering cross section comparable. to that predicted by the parton model. This time is based on the as sumption that the beam has $4 \times 10^{7}$ electrons/pulse at $40 \mathrm{GeV} / \mathrm{c}$.

We propose, therefore, to ask for 200 hours of data-taking time. plus an additional 100 hours, at the same intensity, to checkout and calibrate the counters and to check out the electronics. We will need 100 hours of parasite time to check out the electron and photon beams. 


\section{REFERENCES}

${ }^{1}$ J. D. Bjorken and E. A. Paschos, Phys. Rev. 185, 1975 (1969).

${ }^{2}$ R. P. Feynman, unpublished.

${ }^{3} \mathrm{R}$. Blankenbecler, private communication.

${ }^{4}$ A. Krass, private communication.

${ }^{5}$ L. Caneschi and A. Pignotti, Phys. Rev. Letters 22, 1219 (1969).

${ }^{6}$ J. K. Walker, Proc. Int. Conf. on Expectations for Particle Reactions at the New Accelerators, University of Wisconsin, 1970.

${ }^{7}$ W. T. Toner, Electron and Photon Beams at NAL, National Accelerator Laboratory 1968 Summer Study Report B.9-68-31, Vol. II, p. 125.

${ }^{8}$ R. Diebold and L. Hand, Electron-Photon Beam at NAL, National Accelerator Laboratory 1969 Summer Study Report SS-49, Vol. I, p. 153.

9. O. Caldwell, V. B. Elings, W. P. Hesse, G. E. Jahn, R. J. Morrison, F. V. Murphy, and D. E. Yount, Phys. Rev. Letters 23, $1256(1969)$. 


\section{$-15-$}

\section{FIGURE CAPTIONS}

Fig. 1. Inelastic electron scattering data in the form of a plot of $v W_{2}\left(q^{2}, v\right)$ as a function of $v / q^{2 *}$.

Fig. 2. Calculated photon yields for background ( ${ }^{\circ}$ decays) and signal (inelastic Compton scattering using the parton model) for an incident 20-GeV bremsstrahlung beam.

Fig. 3. The same as Fig. 2 but for a $40-\mathrm{GeV}$ bremsstrahlung beam.

Fig. 4. Layout of the experimental equipment. The drawing is not to scale, but some dimensions are indicated in the figure.

Fig. 5. Electron yield expected for the modified 3.5-mrad beam. The yield is for $10^{13}$ interacting protons/pulse and for a momentum bite $\Delta \mathrm{p} / \mathrm{p}$ of $5 \%$

Fig. 6. The expected statistical errors for data collected in 5 of the 40 lead-glass counters in 200 hours of running. Three curves are shown: 1) $\pi^{\circ}$ decay, 2) $\pi^{\circ}$ decay plus inelastic Compton scattering calculated with the parton model using $Q=1,3)$ the same as 2 but with $\left\langle Q^{4}\right\rangle /\left\langle Q^{2}\right\rangle=1 / 3$. 


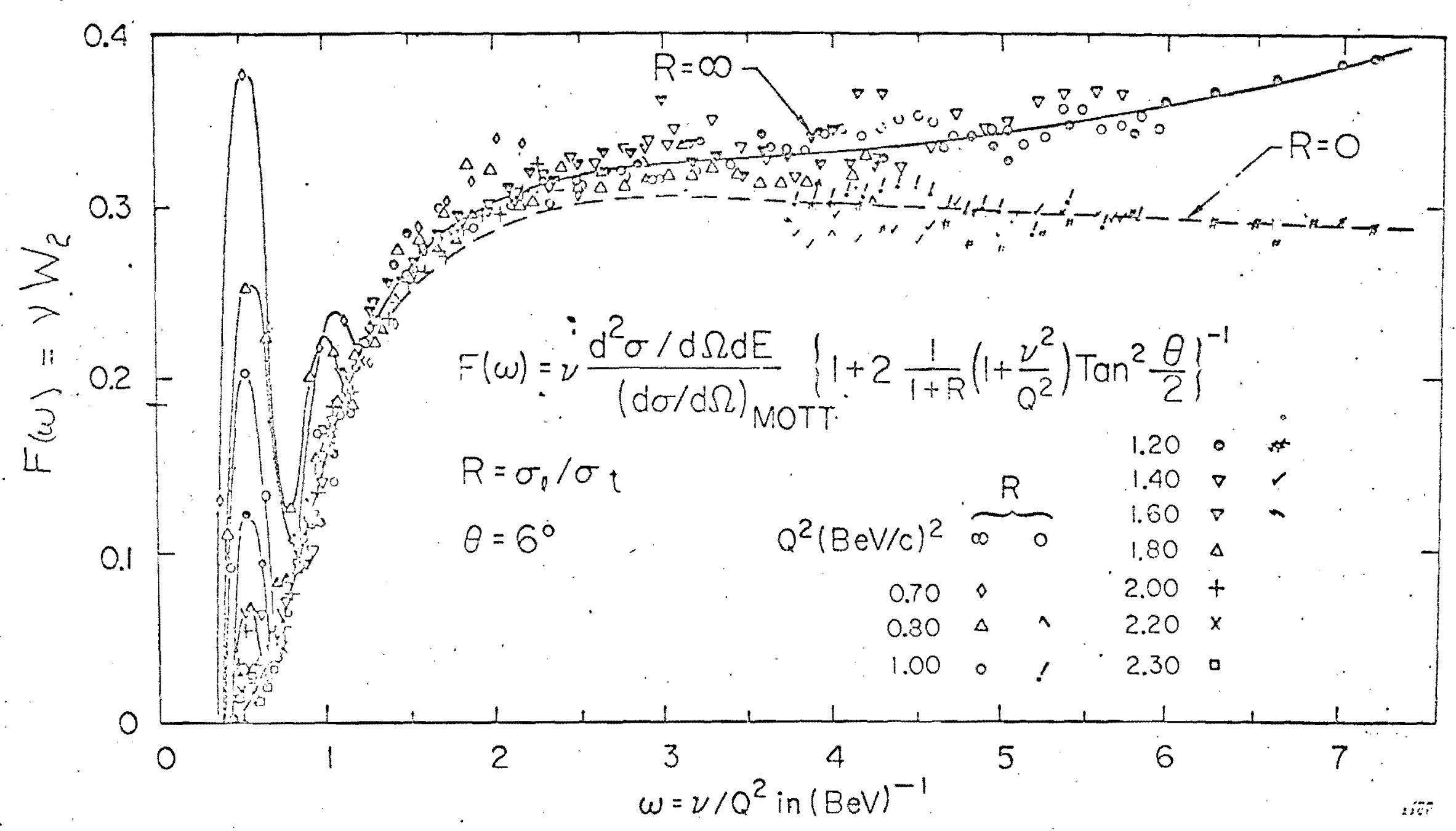

Fig. 1 


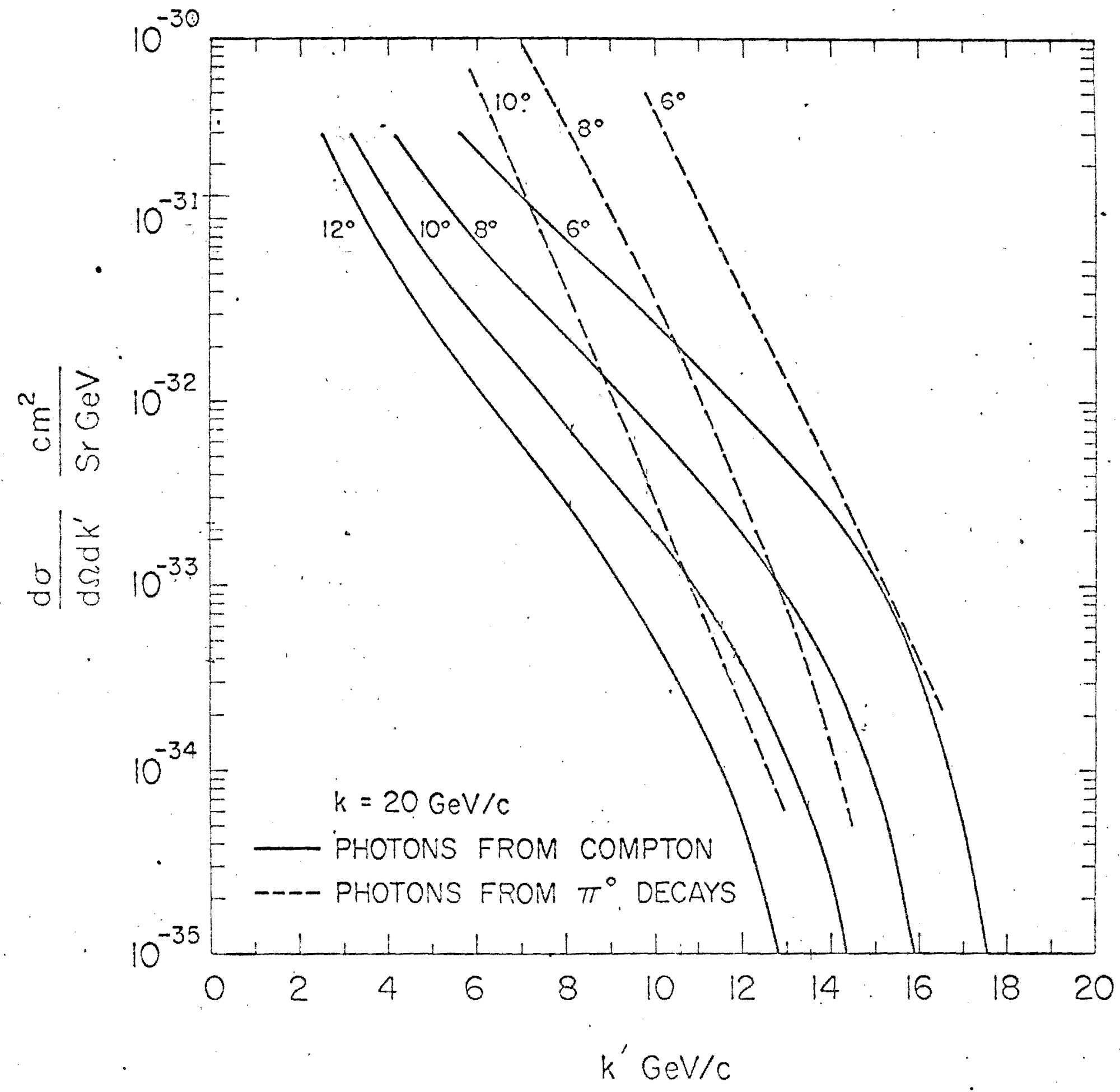


Side Vien

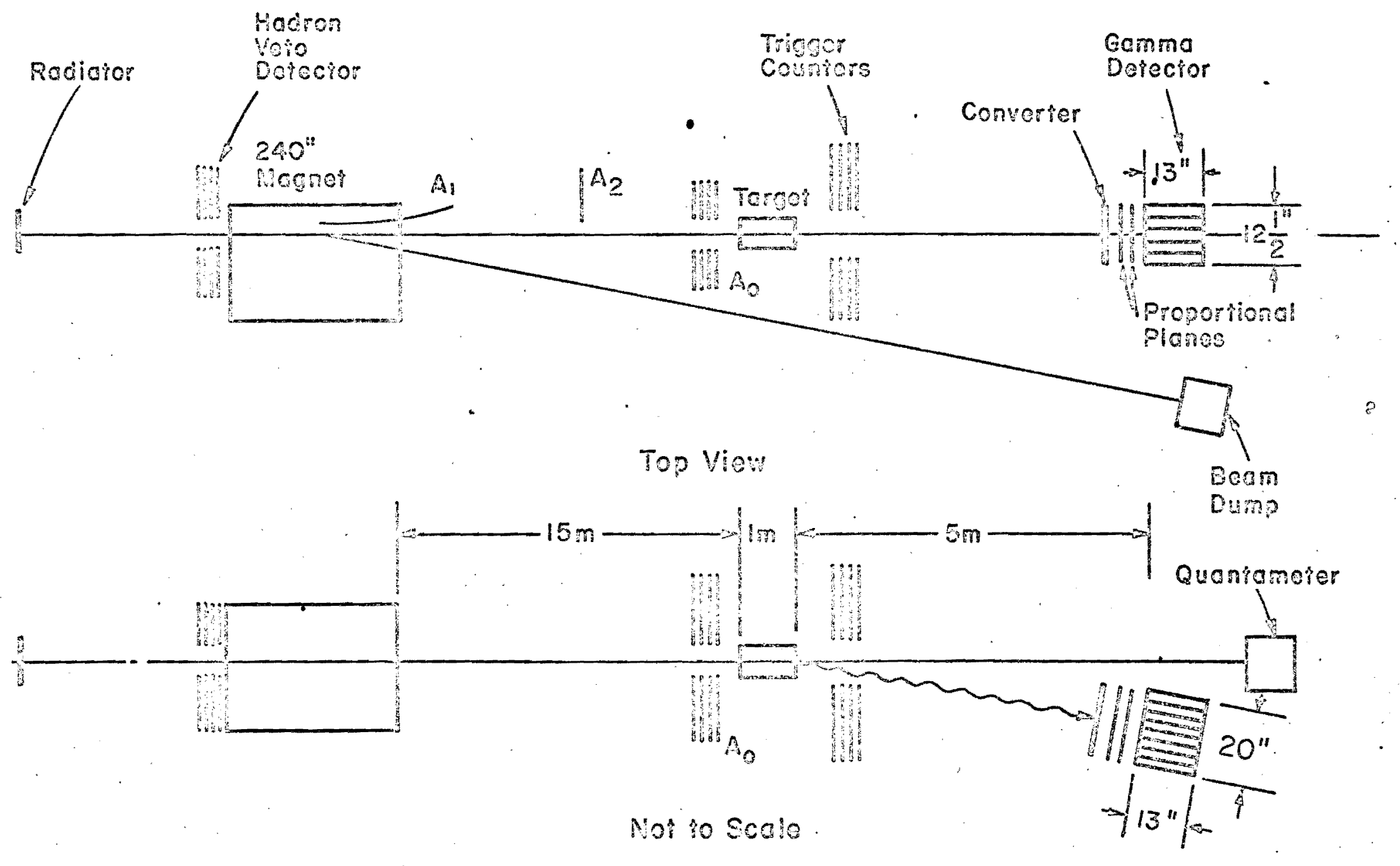

Figuro 4 


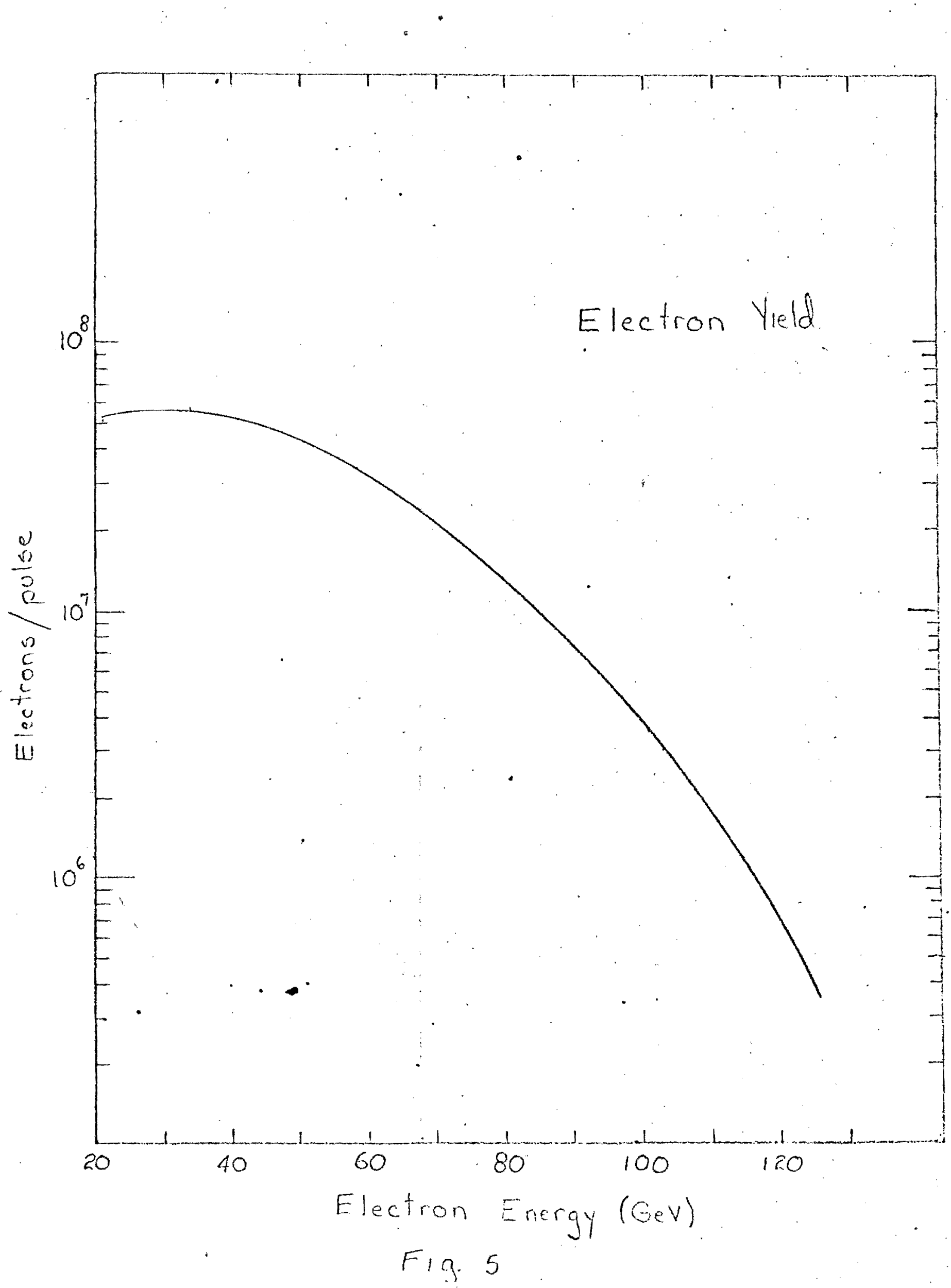


SUPPLEMENT TO NAL PROPOSAL NO. 24

In our original proposal apparently we did not make clear the reason for doing inelastic compton scattering at NAL, as opposed to other accelerators. The reason, which we shall explain more fully below, is basically the signal-to-background ratio. By utilizing bremsstrahlung of $40 \mathrm{GeV}$ instead of $20 \mathrm{GeV}$ the signal-to-background ratio improves by about two orders of magnitude. This permits not only the unambiguous observation of an effect, if photons scatter from point-like constituents of the proton, but also allows the observation of the effect over a sufficient range of secondary photon energies and angles that positive identification of the source of the effect appears possible. The motivation for the experiment arises from the explanation of deep inelastic electron scattering in terms of the interaction of the electrons with point-like constituents of the proton. Such a picture explains in a compelling way the large cross section which decreases remarkably slowly with momentum transfer and which displays "scaling". Since our proposal was written similar scattering has been observed from the neutron, and it is also consistent with this picture. If this point of view is correct, then quite independent of the details of the model, there is a direct proportionality between deep inelastic photon scattering and deep inelastic electron scattering. Aside from kinematic factors, the proportionality constant involves just the charge of the point-like object which is doing the scattering. Thus 
point by point in secondary particle energy and angle the photon cross section is predicted by the electron results, with the normalization giving the charge of the scatterer.

The problem with the experiment is that processes other than inelastic compton scattering can produce high energy photons. Particularly troublesome is the production of high energy $\pi^{\circ}$ 's, which give high energy photons upon decaying. At $20 \mathrm{GeV}$, except at the very highest secondary photon energies, the $\gamma$-rays from $\pi^{\circ}$ decay swamp any signal from inelastic compton scattering. As the incident energy is increased, the $\pi^{\circ}$ background does not change appreciably, but the inelastic compton signal, which -like the inelastic electron scattering -- does not fall off much with momentum transfer, appears more and more clearly as larger secondary photon energies become kinematically available. Thus one can achieve signal-to-background ratios two orders of magnitude better at $40 \mathrm{GeV}$ than at $20 \mathrm{GeV}$. This point is illustrated by two figures from our proposal which are attached.

We have investigated other backgrounds and find $\pi^{\circ}$ decay the worst problem. In particular it has been suggested that $\omega^{\circ} \rightarrow \pi^{\circ}+\gamma$ might be an important background source, particularly because $\omega^{\circ}$ 's are diffractively produced and hence their cross section is energy independent. Detailed calculations show that the contribution of this decay to the high-energy $\gamma^{\prime} s$ at $40 \mathrm{GeV}$ incident energy is negligible. At $20 \mathrm{GeV}$, non-diffractively produced $\omega^{\circ}$ 's are more of a problem, and experimental information is unfortunately sparse, but even this source of $\gamma$-rays appears 
to be significantly less than the $\pi^{\circ} \mathrm{s}$.

Although the data are still being analyzed, there is an

indication from our brief look at $20 \mathrm{GeV}$ inelastic compton scattering at SLAC that high energy $y$ rays are present in roughly the quantity predicted by scattering from point-like constituents of the proton. We can do a better experiment at SLAC, but it is highly probable that only at NAL can a clear signal be observed over a sufficient range of secondary energies and angles to test conclusively the source of these $\gamma$ rays. 


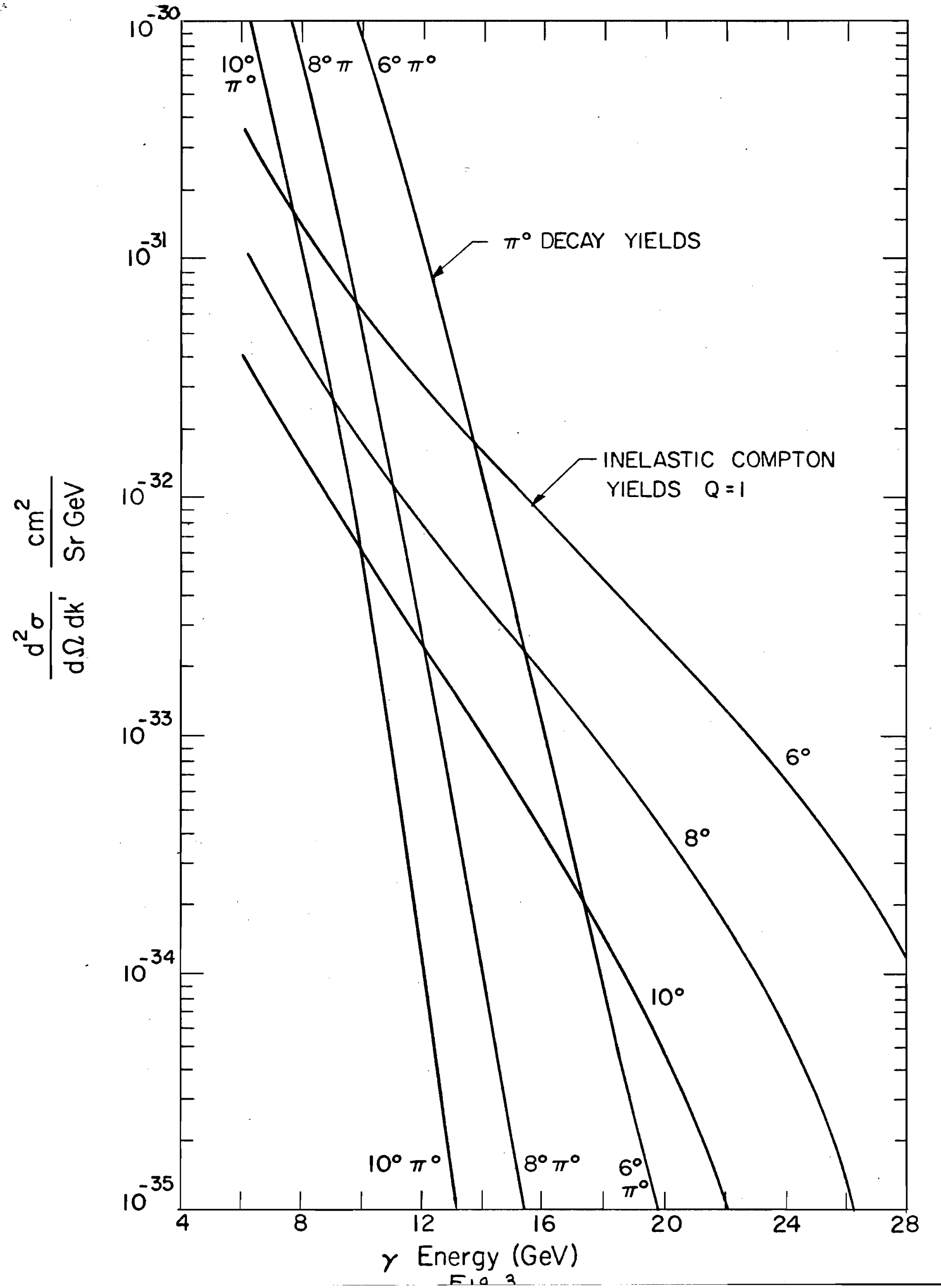




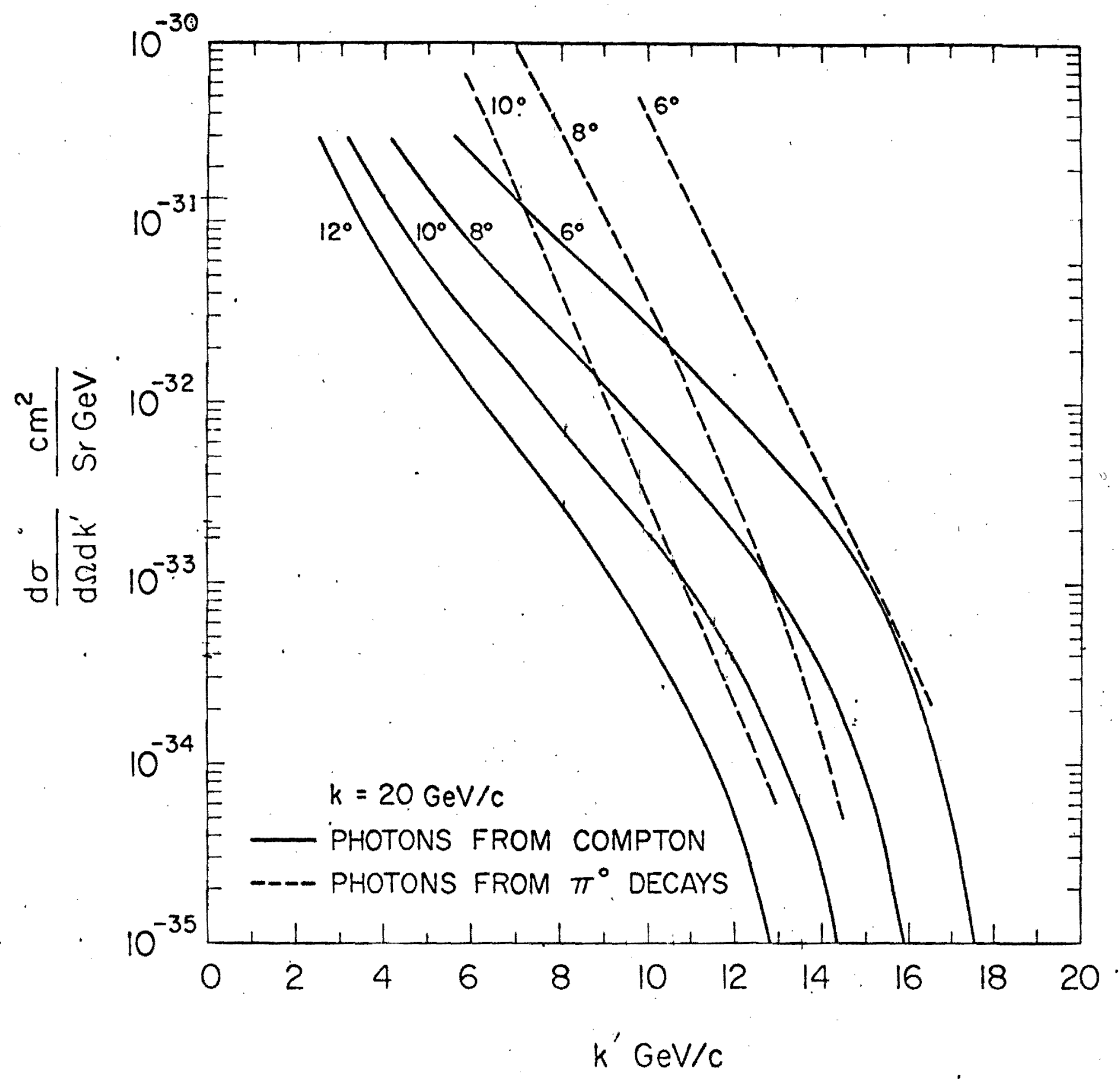

